\title{
A Refined GTD Ray System for an Embedded Object and Its Polarimetric Behavior
}

\author{
Nicolas Pascal Marquart, Member, IEEE, Frédéric Molinet, Member, IEEE, and \\ Eric Pottier, Senior Member, IEEE
}

\begin{abstract}
A refined ray system based on the geometrical theory of diffraction (GTD) for an object embedded in soil for a monostatic transmitter-receiver alignment is presented. Apart from the investigation of the "classical" reflections from the target, creeping waves are also taken into account, and their formalism is presented. The objective of such a ray set is to better understand the different scattering mechanisms, which are presented in the complex scattering framework. In electromagnetic modeling based on GTD, the complex shape of a target is replaced by simpler canonical objects, e.g., facets, cones, wedges, spheres, or cylinders. Here, a cylinder is located in parallel and closely to the plane interface of two dielectric half-spaces. The example of air-soil is taken into account. The numerical results obtained for various directions of incidence are employed to describe the polarimetric characteristics of the diffracted field from grazing to perpendicular incidence to the surface. By representing the diffracted GTD field on the Poincaré sphere, the location on the sphere has a one-to-one relationship to the dielectric properties of the soil. The relation can be employed to extract information as the soil moisture.
\end{abstract}

Index Terms-Creeping waves, geometrical theory of diffraction (GTD), ground penetrating radar (GPR), mine detection, radar polarimetry, soil moisture.

\section{INTRODUCTION}

$\mathbf{S}$ YNTHETICALLY generated data sets are important for radar polarimetry. By forward calculations of individual scattering mechanisms, the entire scattering process can be accurately modeled. A refined ray set is implemented for an object embedded in soil close to the interface. In addition to the multiple reflections of waves between the cylinder and the interface, special emphasis is placed on the creeping waves, encircling the cylinder. In general, the introduced ray system consists of two wave types. The first type describes waves, which propagate in free space after each interaction with the cylinder. They are denoted as "space waves." All multiple reflected waves between the cylinder and the interface are space waves. The second type describes creeping waves and lateral waves. These waves propagate along the surface of the cylinder and the plane interface between two semi-infinite media. They are called "guided waves." By introducing space and guided waves in the ray system, a better understanding of the different

Manuscript received July 9, 2007; revised January 3, 2008.

N. P. Marquart is with the Microwaves and Radar Institute, German Aerospace Center, 82230 Oberpfaffenhofen, Germany.

F. Molinet is with MOTHESIM, 92357 Paris, France.

E. Pottier is with the Institute of Electronics and Telecommunications of Rennes, University of Rennes 1, 35065 Rennes Cedex, France.

Digital Object Identifier 10.1109/TGRS.2008.916634 scattering mechanisms is obtained. In consequence, additional information on the geometrical parameters of the cylinder, its depth, or the dielectric soil properties can be obtained. The latter could be exploited to determine the soil moisture.

From the historical point of view, the diffraction of a plane wave by an object situated close to an interface of a two- or multilayered medium has been investigated by many authors, and extensive literature exists on the subject. By using an integral equation approach, Butler et al. [1] treated the problem of a perfectly conducting cylinder of arbitrary cross section located near the planar interface between two semi-infinite homogeneous half-spaces of different electromagnetic properties and obtained numerical results for the currents induced on a strip, a circular cylinder, and a rectangular cylinder. Michalski and Zheng [2] developed a general procedure for the scattering from perfectly conducting objects of arbitrary shape embedded in a medium consisting of an arbitrary number of planar dielectric layers. The key step in this procedure is the development of a mixed potential integral equation formulation that is amenable to the existing Method of Moments procedure developed for objects in free space. Chang and Mei [3] adapted the analysis of the unimoment method to a partly buried object. All these approaches, which involve the calculation of the half-space Green's function and the evaluation of the Sommerfeld integrals, are limited by the computational and memory requirements when the size of the buried object is large compared to the wavelength. More recently, the computational complexity has been reduced according to Geng and Carin [4] by adapting the fast multipole method to the problem. However, since the interaction between the different parts of a target buried in a lossy half-space depends strongly on the depth and the soil characteristics, the acceleration procedures mentioned in [4] have to be optimized with respect to these parameters. In many particular situations, however, the rigorous treatment of the subsurface diffraction problem by numerical techniques may be unnecessary. Considering the case where the radius $r$ of the buried cylinder is large compared to the wavelength $(r / \lambda \geq 0.64)$, the target-interface interaction can be modeled by a few dominant waves. For solving the diffraction problem, a ray model based on the geometrical optics (GO) and the geometrical theory of diffraction (GTD) has been chosen. The ray model is used to describe the polarimetric behavior of the illuminated target. The polarimetric characteristics can be employed to extract some important physical parameters, such as soil permittivity. In general, the existing coherent or incoherent decomposition theorems of the scattering matrix [s], which may be applied in the polarimetric analysis of the 
problem, consider very simple models based on GO involving single (odd), double (even), or multiple bounce effects (see, e.g., the works of Krogager [5], Cameron [6], or Cloude and Pottier [7]). The polarimetric analysis of the problem is performed by using a more refined ray model composed of spatial and creeping waves. The creeping wave contribution to the diffracted field might be small for some values of the physical parameters (depth and size of the target and characteristics of the soil), but it should not be neglected since it provides a larger domain of application (e.g., the determination of the geometrical properties of a target). The ray set is made up of two spatial waves and three creeping waves in accordance with the GTD and the truncation rule of asymptotic expansions as well. The GTD or étalon method for wave fields states that similar ray geometries lead to similar asymptotic formulas $(k \rightarrow \infty)$. For a smooth convex target in front of an air-soil interface, the simplest étalon problem includes a sphere or cylinder. The solution of the étalon problem gives the main features of the polarimetric behavior of an arbitrary convex target. Exemplarily, we investigate a cylinder embedded in the soil close to the interface. The diffracted ray field for a monostatic alignment of the transceiver antenna is numerically calculated for an incidence angle $\varphi_{i}$ varying from perpendicular $\varphi_{i}=0^{\circ}$ (nadir) to grazing incidence $\varphi_{i}=90^{\circ}$ to the surface. The propagation of these creeping waves over the surface causes radiation of elementary space waves tangentially away from the surface toward the interface. In turn, these space waves generate lateral propagating waves along the interface. The numerical results obtained by the introduced GTD ray system are exploited to investigate the polarimetric characteristics of the diffracted field over the entire incidence angle range.

The structure of this paper is as follows: In Section II, the spatial divergence of a general tube of rays at the transition from the soil to the air is derived. The transition of a wave is described by the coefficient $\mathcal{D}\left(n, \varphi_{i}\right)$. The transition coefficient $\mathcal{D}\left(n, \varphi_{i}\right)$ depends on the incidence angle $\varphi_{i}$ and on the complex refractive index $n$. The refractive index $n$ is determined by the dielectric properties of the soil. Afterward, we present the different wave types of the GTD ray system and discuss their numerical implementation. The contributions of the different wave types are presented in Section III. Based on the numerical results, the polarimetric signature of the diffracted field on the Poincaré sphere over the entire look angle range $0^{\circ}<\varphi_{i}<90^{\circ}$ is derived in Section IV for different soils. Typical examples of trajectories on the Poincaré sphere will be presented showing the correspondence between the characteristics of these trajectories and the soil's permittivity. Final remarks are provided in Section V.

\section{GTD RAY SySTEM}

In the following, a GTD ray field scattered from an embedded circular cylinder is built up by five different waves for a monostatic transmitting-receiving antenna alignment. The antenna is assumed to be located at the point $M$ far away from the cylinder. The corresponding ray paths for the monostatic case are found by applying the principle of Fermat, which dictates that the ray paths follow the minimal temporal trajectory time between two points. In a homogenous medium, Fermat's

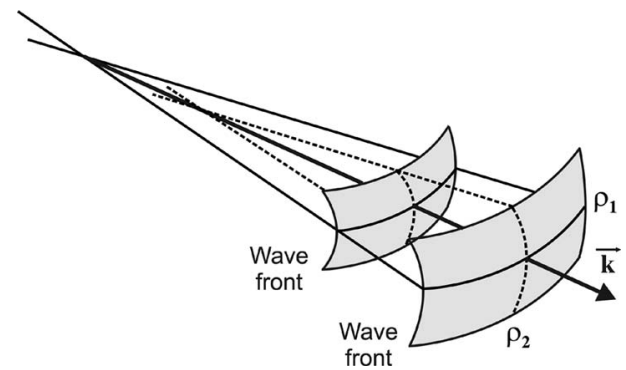

Fig. 1. Tube of rays and its two principal radii $\rho_{1}$ and $\rho_{2}$ of the wave fronts.

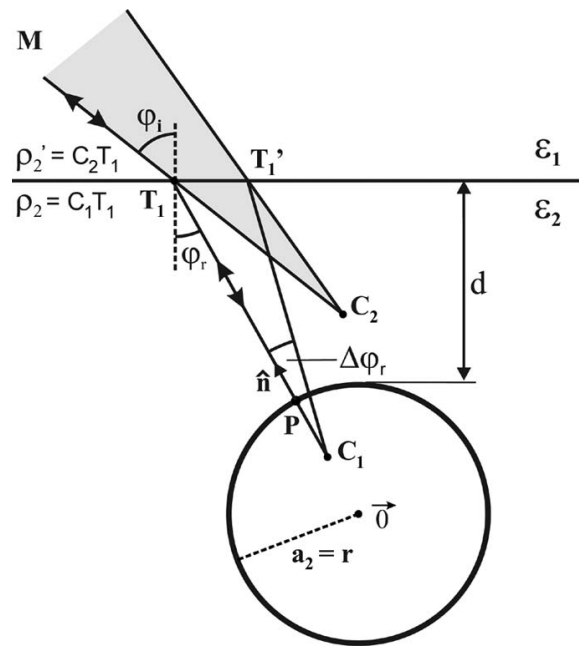

Fig. 2. Expansion of a tube of rays at the transition from the soil $\overline{C_{1} T_{1} T_{1}^{\prime}}$ into the air $\overline{C_{2} T_{1} T_{1}^{\prime}}$.

principle implies that the rays are straight lines. Other basic postulates are that the polarization is constant along such a ray for an isotropic medium and that the power in a flux tube is conserved. Such a tube comprises a bundle of rays, and with the propagation of the bundle tube, the energy diverges. At the transition from the soil to the air, such a tube of rays is spread.

\section{A. Divergence at the Transition}

According to the law of GO, the spatial propagation of a ray and its corresponding energy, which is proportional to the square of the amplitude of the field, is conserved in a tube of rays. The wave front of such a tube is described by two principal radii of curvature in accordance with the differential geometry, as shown in Fig. 1, according to McNamara et al. [8]. In the case of an interaction with an object, the two wave-front curvatures are usually modified and described by a spatial divergence term. An additional modification of the backscattered wave front occurs at the transition from the soil into the air. The transition from a "denser dielectric" half-space into the air leads to a spreading of the tube. The additional geometrical attenuation of the field is described by a general modification coefficient $\mathcal{D}\left(n, \varphi_{i}\right)$. In general, the plane of incidence is determined by the incident wave vector $\vec{k}$ and the local normal vector $\hat{n}$ at the local reflection point. With reference to Fig. 2, a perfectly conducting cylinder embedded in the soil is assumed with its axis oriented parallel to the interface. In that particular case, the section of the cylinder coincides with the plane of incidence. 
Hence, the two principal radii of curvature $\rho_{1}$ and $\rho_{2}$ are given by the perpendicular- and parallel-aligned components relative to the plane of incidence. The modifications of the two principal radii of curvature $\rho_{1}$ and $\rho_{2}$ after a reflection are given with reference to [8] by

$$
\begin{aligned}
& \perp: \quad \frac{1}{\rho_{1}}=\frac{1}{\rho_{1}^{i}}+\frac{2 \cos \left(\varphi_{i}\right)}{a_{1}} \\
& \|: \quad \frac{1}{\rho_{2}}=\frac{1}{\rho_{2}^{i}}+\frac{2}{a_{2} \cos \left(\varphi_{i}\right)}
\end{aligned}
$$

where the local radii $a_{1}$ and $a_{2}$ describe the local surface curvatures at the reflection point and the index $(i)$ signifies the incident wave field. For the cylinder model, this means that the perpendicular radius of curvature $a_{1}$ is equal to infinity. Hence, the perpendicular component of the wave front will not be modified after a reflection on the cylinder. In contrast, the component $a_{2}$ in the plane of incidence will be modified according to (2). In the case of a circular cylinder with radius $r$, the local radius of curvature $a_{2}$ is constant. Furthermore, the angle $\varphi_{i}$ defines the incidence angle between the wave vector $\vec{k}$ and the local normal vector $\hat{n}$. Referring to Fig. 2, the perpendicular radius of curvature $a_{1}$ of the circular cylinder is equal to infinity, and the second radius $a_{2}$ is constant along the circle. As a result, the curvature $\rho_{1}$ of the wave front remains unmodified after a reflection at the cylinder. Hence, in the following, only the radius of curvature lying in the plane of incidence $\rho_{2}$ has to be considered. The reflection and transition of such a single ray are shown in Fig. 2, whereby a plane wave is propagating from the antenna toward the point $T_{1}$ located on the plane interface. After the transition into the soil, the wave is perpendicularly reflected $\cos \left(\varphi_{i}\right)=0^{\circ}$ at the point $P$. After the interaction with the cylinder, the wave propagates along the same path back to the antenna. The round trip of the wave is shown by arrows. According to (2), the modified radius of curvature is given in these studies by $\rho_{2}=a_{2} / 2$ for an incoming plane wave field $\rho_{2}^{i} \rightarrow \infty$. The corresponding caustic is marked by the point $C_{1}$, lying at the half-distance of the path $\overline{0 P}$. Of special interest is the transition of the backscattered wave and its tube of rays at the interface. Regarding a neighbor ray $\overline{C_{1} T_{1}^{\prime}}$ within the tube with the variation $\Delta \varphi_{r}$, the tube $\overline{C_{1} T_{1} T_{1}^{\prime}}$ is altered into the gray-colored tube $\overline{C_{2} T_{1} T_{1}^{\prime}}$ at the transition into the air. We find the following relations for the two ray tube segments with the radii of curvature $\rho_{2}\left(T_{1}\right)=\overline{C_{1} T_{1}}$ and $\rho_{2}^{\prime}\left(T_{1}\right)=\overline{C_{2} T_{1}}$ as follows:

$$
\begin{aligned}
& \overline{C_{1} T_{1}}-\overline{C_{1} T_{1}^{\prime}}=\overline{T_{1} T_{1}^{\prime}} \sin \left(\varphi_{r}\right) \\
& \overline{C_{2} T_{1}}-\overline{C_{2} T_{1}^{\prime}}=\overline{T_{1} T_{1}^{\prime}} \sin \left(\varphi_{i}\right) .
\end{aligned}
$$

In (3) and (4), the approximation $\cos \left(\Delta \varphi_{i}\right) \approx \cos \left(\Delta \varphi_{r}\right) \approx 1$ is calculated due to small variations of the incidence and refraction angles $\Delta \varphi_{i}$ and $\Delta \varphi_{r}$. With reference to the sine rule, it yields for the triangles $\overline{T_{1} C_{1} T_{1}^{\prime}}$ and $\overline{T_{1} C_{2} T_{1}^{\prime}}$ the following expressions:

$$
\begin{aligned}
\frac{\overline{T_{1} T_{1}^{\prime}}}{\sin \Delta \varphi_{r}} & =\frac{\overline{C_{1} T_{1}^{\prime}}}{\overline{\cos \varphi_{r}}} \\
\frac{\overline{T_{1} T_{1}^{\prime}}}{\sin \Delta \varphi_{i}} & =\frac{\overline{C_{2} T_{1}^{\prime}}}{\cos \varphi_{i}} .
\end{aligned}
$$

Substituting (5) in (3) and (6) in (4), we can rewrite

$$
\begin{aligned}
& \overline{C_{1} T_{1}}=\overline{T_{1} T_{1}^{\prime}}\left[\frac{\sin \left(\varphi_{r}\right) \sin \left(\Delta \varphi_{r}\right)+\cos \left(\varphi_{r}\right)}{\sin \left(\Delta \varphi_{r}\right)}\right] \\
& \overline{C_{2} T_{1}}=\overline{T_{1} T_{1}^{\prime}}\left[\frac{\sin \left(\varphi_{i}\right) \sin \left(\Delta \varphi_{i}\right)+\cos \left(\varphi_{i}\right)}{\sin \left(\Delta \varphi_{i}\right)}\right] .
\end{aligned}
$$

Dividing (8) by (7), one gets the following expression:

$$
\begin{aligned}
\frac{\overline{C_{2} T_{1}}}{\overline{C_{1} T_{1}}} & =\frac{\rho_{2}^{\prime}\left(T_{1}\right)}{\rho_{2}\left(T_{1}\right)} \\
& =\left[\frac{\sin \left(\varphi_{i}\right) \sin \left(\Delta \varphi_{i}\right)+\cos \left(\varphi_{i}\right)}{\sin \left(\varphi_{r}\right) \sin \left(\Delta \varphi_{r}\right)+\cos \left(\varphi_{r}\right)}\right]\left[\frac{\sin \left(\Delta \varphi_{r}\right)}{\sin \left(\Delta \varphi_{i}\right)}\right] .
\end{aligned}
$$

Next, a simplification of (9) is performed. Generally, the variation of the angles $\varphi_{r}$ and $\varphi_{i}$ is related via the law of refraction. The transition between the soil and the air is given by

$$
\begin{aligned}
\sin \left(\varphi_{i}\right) & =n \sin \varphi_{r} \\
\sin \left(\varphi_{i}-\Delta \varphi_{i}\right) & =n \sin \left(\varphi_{r}-\Delta \varphi_{r}\right) .
\end{aligned}
$$

The refractive index $n$ is given by $n=\sqrt{\epsilon_{2} / \epsilon_{1}}$. The latter expression (11) is rewritten in the following form:

$$
\begin{aligned}
& \sin \left(\varphi_{i}\right) \cos \left(\Delta \varphi_{i}\right)-\cos \left(\varphi_{i}\right) \sin \left(\Delta \varphi_{i}\right) \\
& =n\left[\sin \left(\varphi_{r}\right) \cos \left(\Delta \varphi_{r}\right)-\cos \left(\varphi_{r}\right) \sin \left(\Delta \varphi_{r}\right)\right] .
\end{aligned}
$$

Assuming a small variation of the angles $\Delta \varphi_{i} \approx \Delta \varphi_{r} \approx 0$ as given for neighbor rays, the sine terms can be neglected in the analysis. In consequence, the relation (12) reduces to the following approximate form:

$$
\frac{\sin \left(\Delta \varphi_{r}\right)}{\sin \left(\Delta \varphi_{i}\right)}=\frac{1}{n} \frac{\cos \left(\varphi_{i}\right)}{\cos \left(\varphi_{r}\right)}
$$

Substituting (13) in (9), for $\Delta \varphi_{i} \approx \Delta \varphi_{r} \approx 0$, and after some algebra, the final expression for the modified radius of curvature is obtained as

$$
\rho_{2}^{\prime}\left(T_{1}\right)=\mathcal{D}\left(n, \varphi_{i}\right) \rho_{2}\left(T_{1}\right)
$$

where the modification coefficient $\mathcal{D}\left(n, \varphi_{i}\right)$ takes the simple form

$$
\mathcal{D}\left(n, \varphi_{i}\right)=\frac{1}{n}\left[\frac{\cos ^{2} \varphi_{i}}{\cos ^{2} \varphi_{r}}\right] .
$$

The energy flow within a tube of rays for a transition from the soil into the air (15) is finally determined by the incidence angle $\varphi_{i}$ and the refractive index $n$.

\section{B. Wave Types}

The formulas of the different waves of the system are introduced. The numerical implementation is performed in such a way that the backscattered field can be computed for a large incidence angle range $0^{\circ}<\varphi_{i}<90^{\circ}$. The code respects the more general case of an elliptical cylinder having two different principal axis; for the sake of simplicity, a circular cylinder is discussed. For convenience, a time dependence of the form 


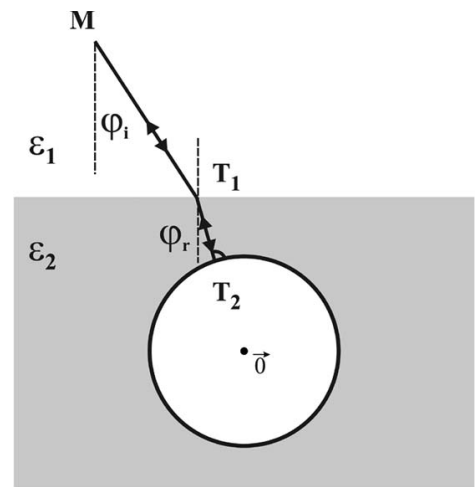

Fig. 3. Ray path of wave 1 .

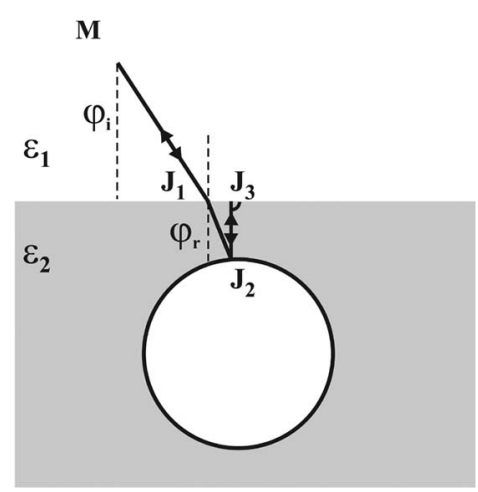

Fig. 4. Ray path of wave 2 .

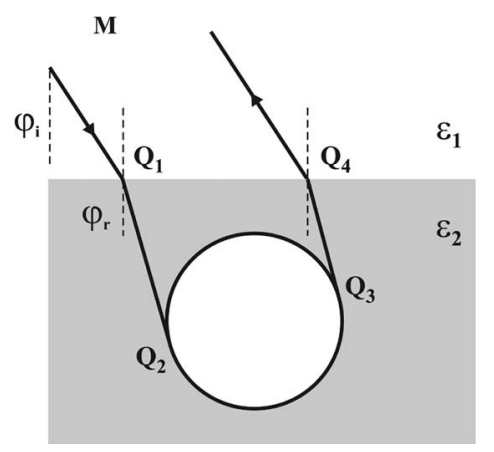

Fig. 5. Ray path of the creeping wave 3.

$\exp (i \omega t)$ is assumed in the following. The path geometries of the various waves are shown in Figs. 3-7. Throughout the calculations, the phase origin is placed in the center of the cylinder, as shown in Fig. 3. Also, for numerical purposes, the spatial divergence term $1 / \sqrt{O M}$ and the phase term $\exp \left(-2 i k_{1} 0 M\right)$ are neglected for each single wave type. The ray geometry and the formulas of the implemented GTD code for the waves 1-5 are discussed next.

1) Wave 1: The first wave type in the system is the one that, after the transition at the interface $T_{1}$, falls perpendicular onto the cylinder $T_{2}$ and is reflected back toward the antenna $M$. The paths for the incoming and backscattered wave are identical. The path geometry and the corresponding interaction points of wave 1 are shown in Fig. 3. The GTD field is computed according to a "brick-building approach," where a simple multiplication of an amplitude term, spreading factor, and phase term describes the propagation of a wave. In accordance with

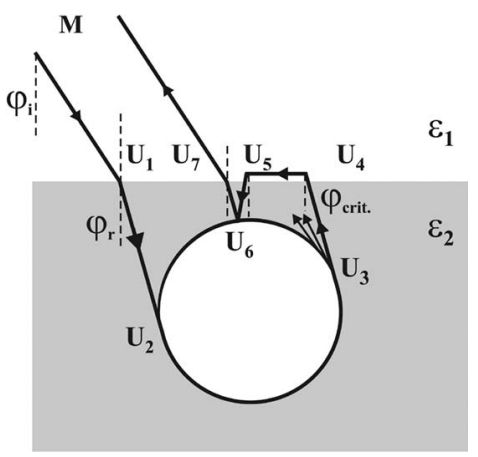

Fig. 6. Ray path of the refracted wave 4.

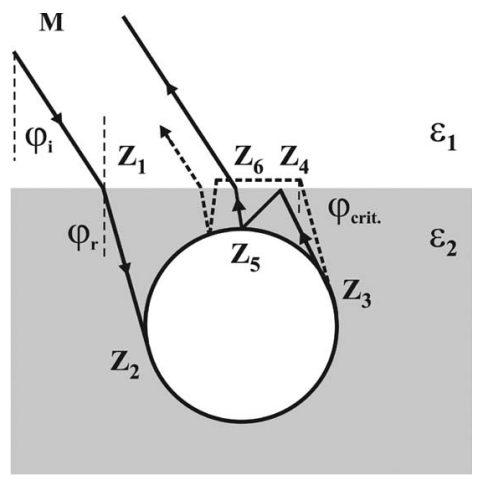

Fig. 7. Ray path of wave 5.

the notation used by Marquart [9], the direct reflected wave 1 from the cylinder is immediately expressed by the following building blocks:

$$
\begin{aligned}
\vec{E}(M)=\vec{E}_{0} \cdot \underline{T}^{i}\left(T_{1}\right) \cdot \underline{R}_{0}\left(T_{2}\right) & \\
& \cdot \underline{T}^{r}\left(T_{1}\right) \mathrm{SF}_{W 1}\left(T_{1}\right) \cdot e^{-2 i\left[\vec{k}_{1}^{i} \cdot \overrightarrow{0 T_{1}}+k_{2} T_{1} T_{2}\right]}
\end{aligned}
$$

where the dyads $\underline{T}^{i}$ and $\underline{T}^{r}$ describe the two transitions of wave 1 across the interface. The dyad $\underline{R}_{0}$ signifies the reflection at $T_{2}$ for the perfectly conducting cylinder. According to (14), the spatial divergence of wave 1 after its transition into the air is given in [8]

$$
\mathrm{SF}_{W 1}\left(T_{1}\right)=\sqrt{\rho_{2}^{\prime}\left(T_{1}\right)}
$$

The change of the radius of curvature $\rho_{2}\left(T_{1}\right)$ after the transition into the air is given explicitly in the following form after (14):

$$
\rho_{2}^{\prime}\left(T_{1}\right)=\mathcal{D}_{\left(n, \varphi_{i}\right)}\left(\frac{a o\left(T_{2}\right)}{2}+T 1 T 2\right)
$$

The divergence factor $1 / \sqrt{\rho_{2}^{\prime}\left(T_{1}\right)+T_{1} M}$ is equated to $1 / \sqrt{0 M}$ and neglected.

2) Wave 2: The second wave discussed describes the first interaction between the target and the interface. It will be named wave 2 in the ray system. Its geometry and interaction points are shown in Fig. 4. In comparison to wave 1, two additional 
reflections, one at the interface $J_{3}$ and one at the cylinder $J_{2}$, are present. The additional interactions signify an attenuation of the field. Similar to wave 1 , the backscattered wave 2 received at the antenna is given by the following blocks:

$$
\begin{array}{r}
\vec{E}(M)=\vec{E}_{0} \cdot \underline{T}^{i}\left(J_{1}\right) \cdot \underline{R}_{0}^{2}\left(J_{2}\right) \cdot \underline{f}\left(J_{3}\right) \cdot \underline{T}^{r}\left(J_{1}\right) \mathrm{SF}_{W 2}\left(J_{1}\right) \\
\cdot e^{-2 i\left[\vec{k}_{1}^{i} \cdot \overrightarrow{0 J_{1}}+k_{2}\left(J_{1} J_{2}+J_{2} J_{3}\right)\right]}
\end{array}
$$

where the Fresnel dyad $f$ takes into account the reflection at the interface. In (19), the radius of curvature at the transition point $J_{1}$ is modified in accordance with (14). When five and more interactions come into play, the received energy at the antenna is negligible. Therefore, the investigation of waves of higher order is meaningless and not included in the ray system.

3) Wave 3: The waves 1 and 2 presented earlier are space waves that do not attach to the cylinder. In contrast, the third wave introduced in the system is the so-called creeping wave, which surrounds the cylinder. The creeping wave 3 and its propagation are shown in Fig. 5. After attaching tangentially at the point $Q_{2}$, the wave surrounds the cylinder as a creeping wave before being tangentially radiated away from the surface $Q_{3}$ toward the antenna at $M$. According to the notation used in [10], the creeping wave 3 is given in the following form:

$$
\begin{aligned}
\vec{E}(M)=2 \vec{E}_{0} \cdot \underline{T}\left(Q_{1}\right) \cdot \underline{D}\left(Q_{2}, Q_{3}\right) \\
\cdot \underline{T}\left(Q_{4}\right) e^{i a t t_{h}^{s}} e^{-i\left[\vec{k}_{1}^{i} \cdot 0 \vec{Q}_{1}+\vec{k}_{1}^{i} \cdot 0 \vec{Q}_{4}+k_{2} Q_{2} Q_{3}\right] .}
\end{aligned}
$$

Referring to Fig. 5, wave 3 propagates on an arc around the cylinder. Its asymptotic approximations of the attachment and detachment coefficients are computed according to the work of Bouche et al. [10]. The $\perp$ and $\|$ notations are used for the soft $(s)$ and hard $(h)$ components of the field, as given in (20). For the coefficients, it holds

$$
e^{i a t t_{h}^{s}}=e^{i \gamma \frac{l_{c}}{a}} .
$$

In (21), the parameters $l_{c}$ and $a$ correspond to the arc length and to the local radius of curvature along the propagation path on the cylinder. For the circular cylinder, the radius of curvature $a$ is constant along the entire arc length. The attenuation factor $\gamma$ is given by

$$
\gamma=\left(\frac{k_{2} a}{2}\right)^{\frac{1}{3}} e^{i \frac{\pi}{3}}\left\{\begin{array}{l}
q_{1} \\
\bar{q}_{1}
\end{array}\right\}+O\left[\left(\frac{k_{2} a}{2}\right)^{\frac{-4}{3}}\right]
$$

where the first zeros of the airy functions $q_{1}$ and $\bar{q}_{1}$ correspond to the $\perp$ and $\|$ field components according to Abramowitz and Stegun [11]

$$
q_{1}=2.33811 \quad \bar{q}_{1}=1.01879 .
$$

The propagation direction of the incoming and backscattered wave 3 along the ray is interchangeable. Thus, the double field value of wave 3 is calculated in the GTD ray system. In the same manner as for the previous waves, the divergence term
$1 / \sqrt{Q_{3} Q_{4} \cdot \mathcal{D}\left(n, \varphi_{i}\right)+Q_{4} M}$ for the return path is substituted by $1 / \sqrt{0 M}$ and neglected.

4) Wave 4: As a result of the transition of the backscattered field from the denser dielectric soil into the air, lateral refracted waves are generated along the interface of the two half-spaces. This type of lateral propagating wave is shown in Fig. 6 and designated as wave 4 . The propagation of wave 4 takes place in such a way that, by surrounding the cylinder, elementary waves are continuously launched tangentially from the surface toward the interface (similar to wave 3). For incident angles at the surface that are greater than the so-called critical angle $\varphi_{\text {crit. }}$, refraction occurs. For example, at the point $U_{4}$, wave 4 is refracted and propagates along the interface. The critical angle $\varphi_{\text {crit. }}$ is defined by the refraction angle $\varphi_{r}=\pi / 2$. According to the law of refraction, it follows that

$$
\varphi_{\text {crit. }}=\arcsin \left(\frac{1}{n}\right)=\arcsin \left(\frac{c_{2}}{c_{1}}\right)
$$

where $c_{1}$ and $c_{2}$ correspond to the propagation velocities in the air and the soil. The propagation velocity of the refracted wave 4 is given by $c_{1}$. In terms of the dielectric properties $\epsilon_{1}$ and $\epsilon_{2},(23)$ is rewritten in the following form:

$$
\varphi_{\text {crit. }}=\arcsin \left(\sqrt{\frac{\epsilon_{1}}{\epsilon_{2}}}\right) .
$$

On its way from $U_{4}$ to $U_{5}$, the refracted wave 4 continuously radiates elementary waves into the soil with the critical angle $\varphi_{\text {crit. }}$. The field amplitude is exponentially attenuated with the distance from the interface according to Bouche et al. [10]. Referring to Fig. 6, it is apparent that all the detachment points $U_{3}$ along the cylinder represent an excited line source. The problem consists in finding an exact analytical solution of a line source radiating near the interface between two dielectric halfspaces generating lateral waves along the interface. A solution for a line source in the presence of a semi-infinite dielectric medium is given in the work of Felsen [12]. According to the notation used in Fig. 6 and considering [12, Sec. 5.5, eq. (57c); Fig. 5.5.2(b)], the field of a wave radiating from the point $U_{3}$ and propagating as a lateral wave toward the point $U_{6}$ is given as follows:

$$
I_{b}=\frac{1}{\sqrt{2 \pi}} \frac{e^{i\left[k_{2}\left(\overline{U_{3} U_{4}}+\overline{U_{5} U_{6}}\right)+k_{1} \overline{U_{4} U_{5}}\right]} \epsilon^{\frac{1}{4}} e^{-i \frac{\pi}{4}}}{(1-\epsilon)\left(k_{2} \overline{U_{4} U_{5}}\right)^{\frac{3}{2}}}
$$

whereby the substitution $\epsilon=\epsilon_{1} / \epsilon_{2}$ is made. According to the work of Felsen and Marcuvitz [12], a normalized field strength $\left|E_{0}\right|=1$ is assumed at the detachment point $U_{3}$. In contrast, the field strength mentioned in [10, App. 1 (p. 467)] is defined as

$$
E_{0}\left(U_{3}\right)=\underbrace{\frac{e^{i \frac{\pi}{4}}}{\sqrt{8 \pi k}}}_{D_{s}} \frac{e^{i k r}}{\sqrt{r}} .
$$

Hence, in order to compute the field up to the point $U_{6}$ in accordance with the formulas specified in [12], the field value 
at $U_{3}$ has to be divided by the factor $D_{s}$. This finally yields the correct field value of wave 4 in the ray system as follows:

$$
\begin{aligned}
& \vec{E}(M)=\vec{E}_{0} \underline{T}^{i}\left(U_{1}\right) \cdot \underline{D}\left(U_{2}, U_{3}\right)\left(\frac{I_{b}}{D_{s}}\right) \underline{T}^{r}\left(U_{7}\right) \mathrm{SF}_{W 4} \\
& \cdot e^{-i\left[\vec{k}_{1}^{i} \cdot \overrightarrow{0 U_{1}}+\vec{k}_{1}^{r} \cdot \overrightarrow{0 U_{7}}+k_{2}\left(U_{1} U_{2}+U_{2} U_{3}+U_{6} U_{7}\right)\right]} .
\end{aligned}
$$

In (27), the spatial divergence up to $U_{6}$ is calculated. After the reflection at $U_{6}$, the radius of curvature is modified in the following way:

$$
\rho_{2}\left(U_{6}\right)=\frac{a_{2}\left(U_{6}\right) \cos \left(\varphi_{i}\right)}{2} .
$$

The propagation to the transition point $U_{7}$ yields

$$
\rho_{2}\left(U_{7}\right)=\rho_{2}\left(U_{6}\right)+U_{6} U_{7} .
$$

At $U_{7}$, the radius is changed after the transition according to

$$
\rho_{2}^{\prime}\left(U_{7}\right)=\mathcal{D}\left(n, \varphi_{i}\right) \rho_{2}\left(U_{7}\right) .
$$

Finally, the divergence for the return path takes the following form:

$$
\mathrm{SF}_{W 4}=\sqrt{\rho_{2}^{\prime}\left(U_{7}\right)}
$$

where $1 / \sqrt{\rho_{2}^{\prime}\left(U_{7}\right)+U_{7} M}$ is approximated by $1 / \sqrt{0 M}$.

5) Wave 5: Bearing in mind that wave 4 radiates waves tangentially away from the surface on its path around the cylinder, a bundle of launched rays will have incident angles at the interface smaller than the critical angle $\varphi_{\text {crit. }}$. From the bundle of rays, one single wave will propagate back to the antenna, as shown in Fig. 7. Therefore, one may see wave 5 in the succeeding discussion as a special case of the previous wave 4 . In contrast, wave 5 is not propagating as a refracted wave along the interface. Instead, wave 5 is reflected at the interface $Z_{4}$ and cylinder $Z_{5}$ before propagating back toward the antenna. According to the GTD, wave 5 is computed in the following way:

$$
\begin{aligned}
\vec{E}(M)=\vec{E}_{0} \cdot \underline{T}^{i}\left(Z_{1}\right) & \underline{D}\left(Z_{2}, Z_{3}\right) \cdot \underline{f}\left(Z_{4}\right) \cdot \underline{R}_{0}\left(Z_{5}\right) \\
\cdot & \underline{T}^{r}\left(Z_{6}\right) \mathrm{SF}_{W 5} e^{-i\left[\vec{k}_{1}^{i} \cdot \overrightarrow{0 Z_{1}}+k_{2} Z_{1} Z_{6}\right]}
\end{aligned}
$$

where the term $Z_{1} Z_{6}$ corresponds to the entire ray path $Z_{1} Z_{2}+Z_{2} Z_{3}+Z_{3} Z_{4}+Z_{4} Z_{5}+Z_{5} Z_{6}$. The spatial divergence of a ray tube is computed similar to the previously discussed waves. The radius of curvature $\rho_{2}^{\prime}\left(Z_{5}\right)$ after the reflection at $Z_{5}$ is evaluated as follows:

$$
\rho_{2}^{\prime}\left(Z_{5}\right)=\frac{1}{\frac{1}{Z_{3} Z_{4}+Z_{4} Z_{5}}+\frac{2}{a_{2}\left(Z_{5}\right) \cos \left(\varphi_{i}\right)}} .
$$

The modified curvature $\rho_{2}^{\prime}\left(Z_{6}\right)$ after the transition is found to be

$$
\rho_{2}^{\prime}\left(Z_{6}\right)=\mathcal{D}\left(n, \varphi_{i}\right) \quad\left(\rho_{2}^{\prime}\left(Z_{5}\right)+Z_{5} Z_{6}\right) .
$$

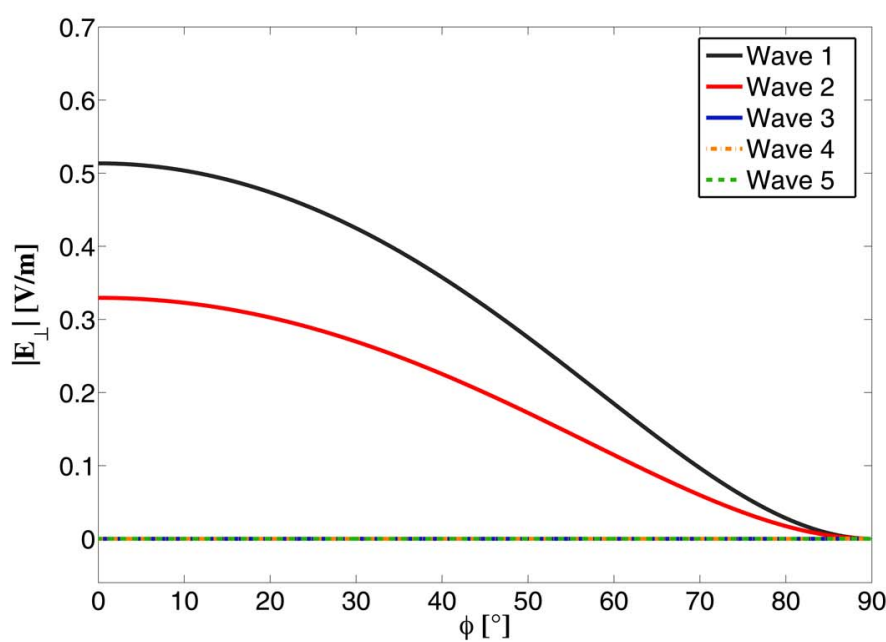

(a)

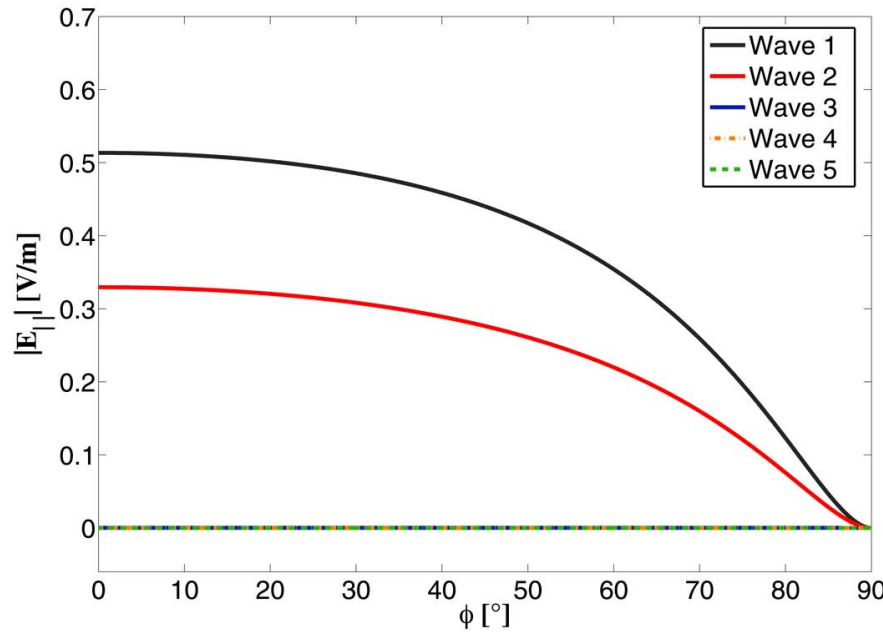

(b)

Fig. 8. Cylinder $(r=2 \mathrm{~m})$ located at the depth $d=0.5 \mathrm{~m}$ in a loss-free soil with $\epsilon_{2}=9.6$. (a) Single-field contributions $\left|\vec{E}_{\perp}(\phi)\right|$ of the ray system. (b) Single-field contributions $\left|\vec{E}_{\|}(\phi)\right|$ of the ray system.

Hence, the spreading factor is given by

$$
\mathrm{SF}_{W 5}=\sqrt{\rho_{2}^{\prime}\left(Z_{6}\right)} .
$$

In the latter expression, the term $1 / \sqrt{\rho_{2}^{\prime}\left(Z_{6}\right)+Z_{6} M}$ is substituted by $1 / \sqrt{0 M}$ and neglected in the field simulations.

\section{Numerical RESULTS}

The backscattered GTD field is computed for a normalized and linearly polarized incident field with the specific field components $\vec{E}_{\perp}^{0}=1, \vec{E}_{\|}^{0}=1$ at the frequency $f=500 \mathrm{MHz}$. The ray system is calculated for a look angle range $\varphi_{i}$ varying from $0^{\circ}$ to $90^{\circ}$. First, the cylinder is placed at a depth $d=0.5 \mathrm{~m}$ in a dielectric lossless soil $\epsilon_{2}=9.6$. The absolute amplitude values of the single waves are shown in Fig. 8 over the entire look angle range. From it, one sees that, due to the strong attenuation of the creeping waves, the dominant contributors of the backscattered ray field are given by the two waves 1 and 2 . The fields of the waves 3,4 , and 5 , which creep on an arc along the cylinder, can be neglected in comparison to the waves 1 


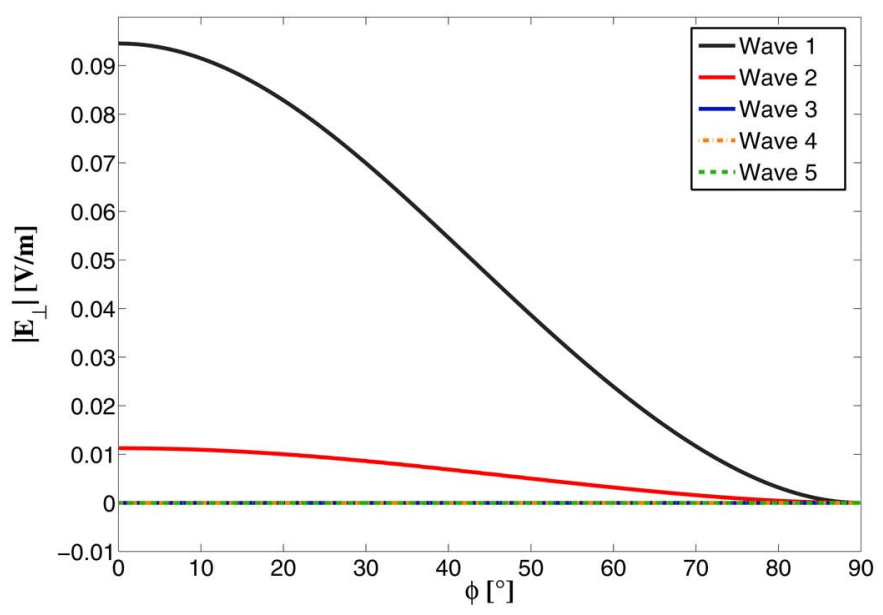

(a)

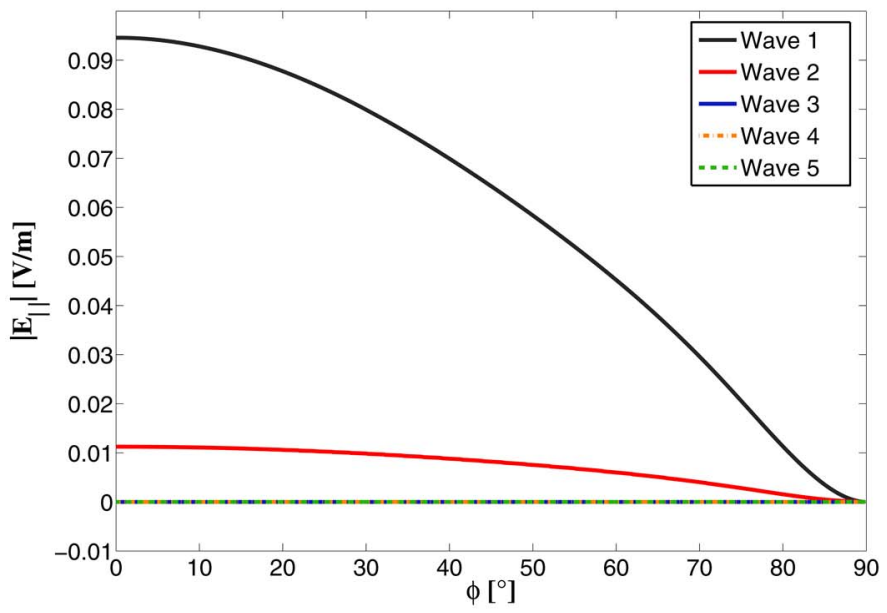

(b)

Fig. 9. Cylinder $(r=2 \mathrm{~m})$ located at the depth $d=0.5 \mathrm{~m}$ in a loss-free soil for a permittivity of $\epsilon_{2}=9.6-i$. (a) Single-field contributions $\left|\vec{E}_{\perp}(\phi)\right|$ of the ray system. (b) Single-field contributions $\left|\vec{E}_{\|}(\phi)\right|$ of the ray system.

and 2. The example shows that the main GTD ray contributors 1 and 2 accurately describe the main polarimetric features according to Marquart et al. [13].

The ray system is computed for a lossy electromagnetic soil where the complex permittivity is set equal to $\epsilon_{2}=9.6-i$. With reference to the work of Ulaby et al. [14], this corresponds, for example, to a sandy soil with poor moisture. In contrast to the loss-free soil, the real refraction angle now becomes imaginary. The formulas can also be applied in that case. With reference to the study of Wang and Deschamps [15], their extension to a lossy medium can be performed as the diffracted field is observed far away from the cylinder. The simulated field values for a lossy soil are shown in Fig. 9 for a complex permittivity $\epsilon_{2}=9.6-i$. In the case that the attenuation is strong, only the main reflection from the cylinder (wave 1) remains as a significant backscattered signal.

\section{Representation on the Poincaré Sphere}

The polarimetric signatures of the backscattered GTD ray field is investigated for an incoming field varying from grazing

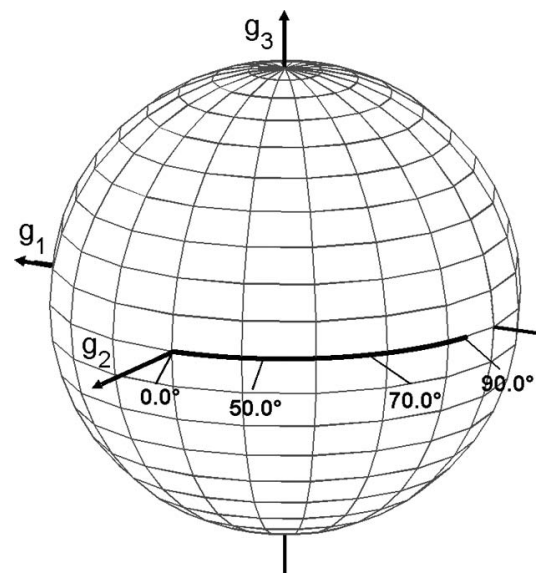

Fig. 10. Polarimetric characteristics of wave 1 over the look angle range $0^{\circ}<$ $\varphi<90^{\circ}$ for a linearly polarized incident field and loss-free soil.

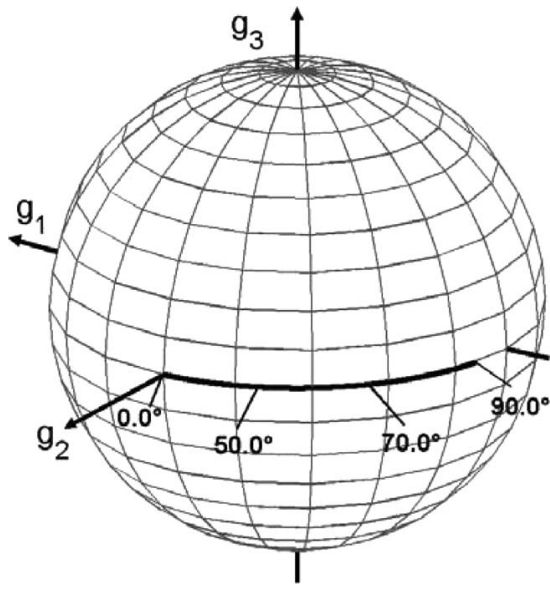

Fig. 11. Polarimetric characteristics of wave 2 over the look angle range $0^{\circ}<$ $\varphi<90^{\circ}$ for a linearly polarized incident field and loss-free soil.

up to perpendicular incidence. The polarimetric behavior is analyzed by representing the components of the Stokes vector $g_{1}, g_{2}$, and $g_{3}$ on a normalized sphere, the Poincaré sphere according to Born and Wolf [16]. Its radius is defined as follows:

$$
g_{0} \leq \sqrt{g_{1}^{2}+g_{2}^{2}+g_{3}^{2}}
$$

The polarization of the two main contributor waves 1 and 2 is represented individually on the Poincaré sphere. A linear polarized incident field given by $\vec{E}_{\perp}^{0}=\vec{E}_{\|}^{0}=1$ at the frequency $f=500 \mathrm{MHz}$ is taken into account. The cylinder is placed in a lossless soil at the depth $d=0.5 \mathrm{~m}$. In Figs. 10 and 11, the polarimetric signatures of waves 1 and 2 are shown over the entire look angle range of $0^{\circ}-90^{\circ}$. According to the Stokes vector and its representation on the Poincaré sphere, the location of the waves 1 and 2 on the sphere is given by $g_{1}=0, g_{2}=1$, and $g_{3}=0$ at perpendicular incidence $\varphi=0^{\circ}$. With increasing look angle, the phase difference of the transition coefficients also increases, leading to the characteristic progression along the equator line toward $g_{1}=-1, g_{2}=0$, and $g_{3}=0$. The transition coefficients of the two field components $\vec{E}_{\perp}^{0}$ and $\vec{E}_{\|}^{0}$ 


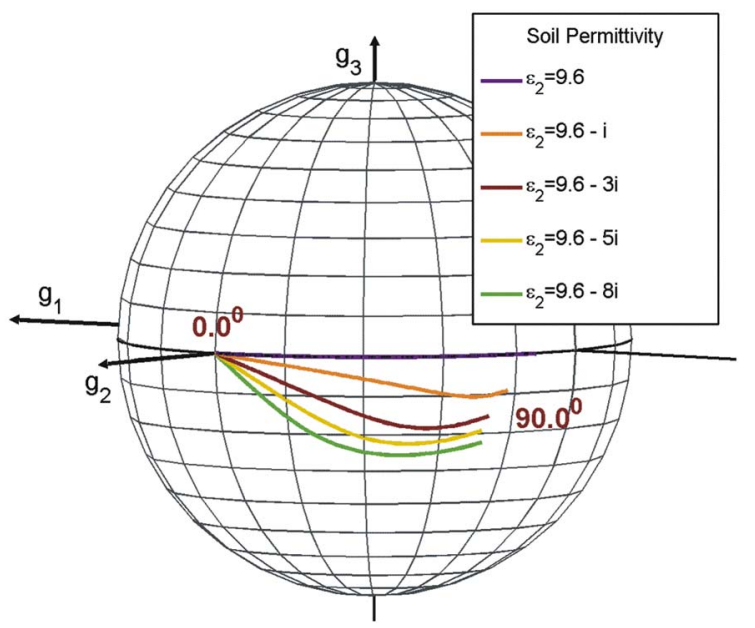

Fig. 12. Polarimetric behavior of the entire ray system over the look angle range of $0^{\circ}-90^{\circ}$ for a linearly polarized incident field. The cylinder is located at the depth $d=0.3 \mathrm{~m}$.

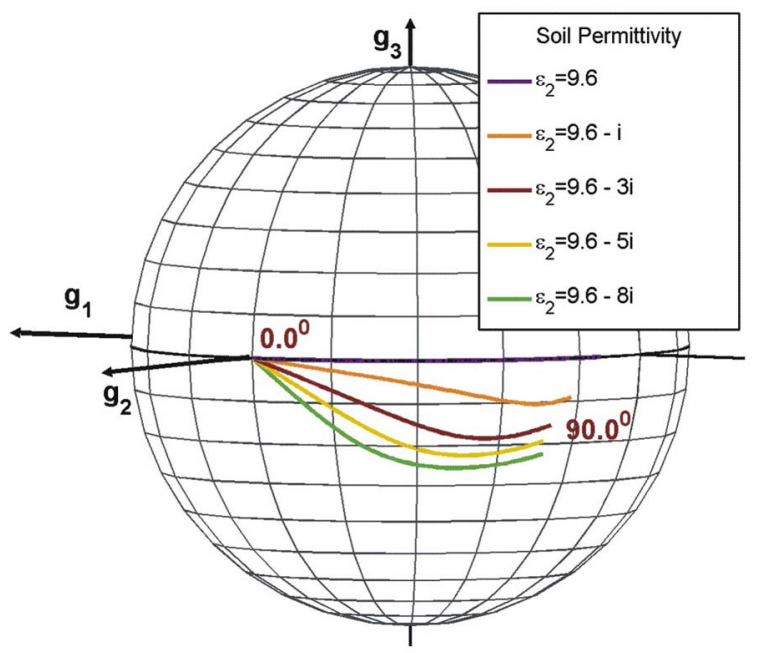

Fig. 13. Polarimetric behavior of the entire ray system over the look angle range of $0^{\circ}-90^{\circ}$ for a linearly polarized incident field. The cylinder is located at the depth $d=0.5 \mathrm{~m}$.

are similar for perpendicular incidence. In order to investigate the effect of the distance between the cylinder and the interface, the backscattered field is calculated at two different depths, namely, $d=0.3 \mathrm{~m}$ and $d=0.5 \mathrm{~m}$. The polarization of the ray field for the different lossy soils is shown in Figs. 12 and 13. With increasing loss, the locations on the sphere move away from the equator line leading to a typical "polarization ladder." Of particular interest is the fact that the superposition of the two main contributors (waves 1 and 2) gives virtually a linear polarization for both computed heights in a loss-free soil. Furthermore, comparing both figures, it is obvious that the distance from the interface has no significant influence on the location on the sphere. Hence, the shift away from the equator line is solely due to the soil's loss. The result is surprising, as one may think that, due to different ray paths, the superposition of the two principal waves should take place out of phase. This is not the case, as due to the geometrical setup and refraction index of the lossy soil, the path length $\overline{J_{1} J_{2}}+\overline{J_{2} J_{3}}$ of wave

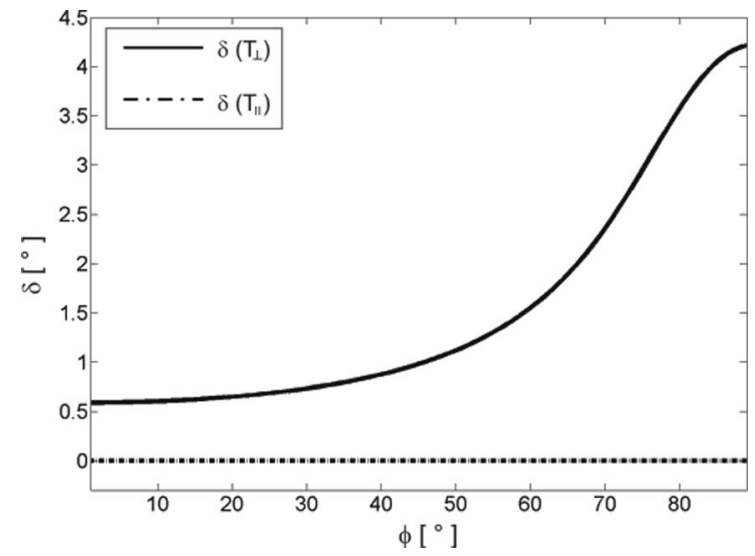

Fig. 14. Absolute phase $\delta$ of the complex transmission coefficients $T_{\perp}$ and $T_{\|}$for a lossy soil: $\epsilon_{2}=9.6-i$.

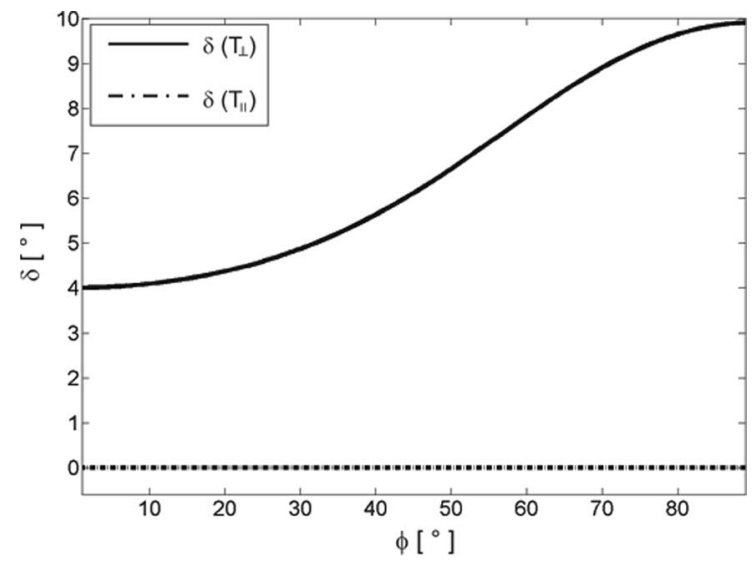

Fig. 15. Absolute phase $\delta$ of the complex transmission coefficients $T_{\perp}$ and $T_{\|}$for a lossy soil: $\epsilon_{2}=9.6-8 i$.

2 is nearly twice as long over the entire look angle range as the distance sphere $\rightarrow$ interface $\overline{T_{1} T_{2}}$ according to wave 1 . As a consequence, the single steps of the polarization ladder can be directly related to the loss of the soil. The small phase shift is given by the transition soil $\rightarrow$ air. For the transition coefficient, a phase shift occurs at the transition lossy soil $\rightarrow$ air. The phases $\delta$ 's of the perpendicular and parallel transmission coefficients are shown in Figs. 14 and 15 for complex permittivities $\epsilon_{2}=9.6-i$ and $\epsilon_{2}=9.6-8 i$. With increasing loss, the phase difference also increases, which finally leads to the significant "polarization ladder" on the Poincaré sphere.

\section{CONCLUSION}

The backscattered field of a cylinder embedded in the soil close to the plane interface is described by a refined ray field for a monostatic transmitter-receiver alignment. The ray system, according to the GTD, is composed of five different waves including space, creeping, and laterally refracted waves along the interface. In contrast to the space waves, the creeping waves attach to the cylinder, surround it, and are again reradiated to the receiving antenna. The ray description of the scattered field via its asymptotic formulas $(k \rightarrow \infty)$ gives a better insight 
into the individual mechanisms of the entire global scattering process. In our example, the waves 1 and 2 remain significant contributors in the ray system. The contribution of the creeping waves is strongly dependent on the frequency of the incident field. The frequency $f=500 \mathrm{MHz}$ is considered, which corresponds to the factor $k r=20$, where $r$ signifies the radius of the circular cylinder. The presented formulas are valid up to the limit $k r=4(f=100 \mathrm{MHz})$ and, thus, applicable for a wider frequency range. Hence, the contributions of the creeping waves to the ray system must be maintained. With decreasing frequency, their contribution to the total field will increase. The implemented GTD formulas are determined for a lossless dielectric medium. Their extension to a lossy medium can be performed for a transceiver antenna located far away from the target. Hence, the two dominant contributors, waves 1 and 2, are a good approximation of the solution. The backscattered GTD field is represented on the Poincare sphere for different lossy soils over the look angle range of $0^{\circ}-90^{\circ}$. The resulting location on the Poincaré sphere provides a one-to-one relation to the dielectric properties of the soil. This could be exploited to determine the soil moisture by burying a reference object and measuring with a ground penetrating radar for instance.

\section{REFERENCES}

[1] C. M. Butler, X. Xiao-Bang, and A. Glisson, "Current induced on a conducting cylinder located near the planar interface between two semiinfinite half spaces," IEEE Trans. Antennas Propag., vol. AP-33, no. 6, pp. 616-624, Jun. 1985.

[2] K. A. Michalski and D. Zheng, "Electromagnetic scattering and radiation by surfaces of arbitrary shape in layered media-Part I: Theory," IEEE Trans. Antennas Propag., vol. 38, no. 3, pp. 335-344, Mar. 1990.

[3] H. S. Chang and K. K. Mei, "Scattering of electromagnetic waves by buried and partly buried bodies of revolution," IEEE Trans. Geosci. Remote Sens., vol. GRS-23, no. 4, pp. 596-605, Jul. 1985.

[4] N. Geng and L. Carin, "Fast multipole method for scattering from an arbitrary PEC target above or buried in a lossy half space," IEEE Trans. Antennas Propag., vol. 49, no. 5, pp. 740-748, May 2001.

[5] E. Krogager, Aspects of Polarimetric Radar Imaging. Copenhagen, Denmark: Danish Defence Res. Establishment, 1993.

[6] W. L. Cameron and L. K. Leung, "Feature motivated polarization scattering matrix decomposition," in Proc. IEEE Int. Radar Conf., Rec., May 1990, pp. 549-557.

[7] S. R. Cloude and E. Pottier, "Concept of polarization entropy in optical scattering," Opt. Eng., vol. 34, no. 6, pp. 1599-1610, Jun. 1995.

[8] D. A. McNamara, C. W. I. Pistorius, and J. A. G. Malherbe, Introduction to the Uniform Geometrical Theory of Diffraction. Boston, MA: Artech House, 1990.

[9] N. P. Marquart, "Investigation on the polarimetric behavior of the emfield scattered by an object located near the interface between the air and a lossy dielectric half space," "Ph.D. dissertation," Univ. Rennes 1, Inst. Electron. Telecommun. Rennes, Rennes Cedex, France, Jan. 2006. [Online]. Available: http://tel.ccsd.cnrs.fr

[10] D. Bouche, F. Molinet, and R. Mittra, Asymptotic Methods in Electromagnetics. Berlin, Germany: Springer-Verlag, 1997.

[11] M. Abramowitz and I. Stegun, Handbook of Mathematical Functions. New York: Dover, 1970.

[12] L. Felsen and N. Marcuvitz, Radiation and Scattering of Waves. Englewood Cliffs, NJ: Prentice-Hall, 1973.

[13] N. P. Marquart, F. Molinet, and E. Pottier, "Investigations on the polarimetric behavior of a target near the soil," IEEE Trans. Geosci. Remote Sens., vol. 44, no. 10, pp. 2899-2907, Oct. 2006.

[14] F. Ulaby, R. Moore, and A. Fung, Microwave Remote Sensing, Active and Passive, vol. III. Boston, MA: Artech House, 1986.

[15] W. D. Wang and G. A. Deschamps, "Application of the complex ray tracing to scattering problems," Proc. IEEE, vol. 62, no. 11, pp. 15411551, Nov. 1974.

[16] M. Born and E. Wolf, Principles of Optics. New York: Pergamon, 1986.

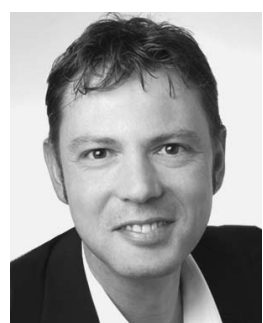

Nicolas Pascal Marquart (M'06) was born in Ehingen/Danube, Germany, on April 26, 1971. He received the Dipl.-Geophys. degree in the area of ground penetrating radar from the University of Karlsruhe, Karlsruhe, Germany, in 2000, and the Ph.D. degree in electromagnetic diffraction theory and radar polarimetry from the University of Rennes 1, Rennes Cedex, France, in 2006.

From 2000 to 2003, he was with MOTHESIM, Paris, France, in the frame of the Training and Mobility program for young researchers of the European Commission. From 2004 to 2005, he was with the Research Training Network of the European Commission and worked in the project Application of Multiparameter Polarimetry in Environmental Remote Sensing with the Institute for the Protection and Security of the Citizen, Joint Research Centre, Ispra, Italy. He is currently with the Microwaves and Radar Institute, German Aerospace Center, Oberpfaffenhofen. His current research activities are centered on HF radar scattering from an ocean surface in the framework of the German Indonesian Tsunami Early Warning System project.

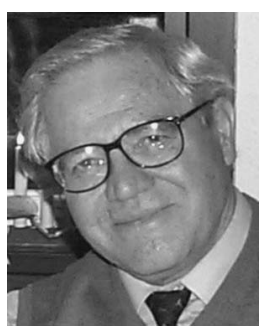

Frédéric Molinet (M'82) was born in Wengelsbach, France, on November 13, 1934. He received the engineering degree from the Ecole Centrale de Lyon, Lyon, France, in 1959, the Doctor de spécialité ( $3^{e}$ cycle) in theoretical nuclear physics from the University of Strasbourg, Strasbourg, France, in 1971, and the Doctor-és-Sciences degree from the University of Paris, Paris, France, in 1971.

From 1964 to 1971 , he was involved in research in plasma physics with the Institut Henri Poincaré, Paris. In 1971, he was with the Laboratoire Central de Télécommunications as a Group Leader with the Department of Theoretical Studies and Modeling. In 1980, he founded MOTHESIM, Paris, where he is currently the Director and has been active in the development of numerical and asymptotic techniques for the electromagnetic and acoustic radiation and scattering problems, with applications to electromagnetic pulse, electromagnetic compatibility radar, and sonar scattering.

Dr. Molinet is a former President of the Waves and Fields chapter of the Sociéte des Electrisiens, Electroniciens et des Radioélectriciens and the Chairman of the French URSI Commission B.

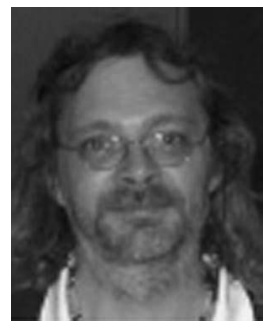

Eric Pottier (M'95-SM'06) received the M.Sc. and $\mathrm{Ph} . \mathrm{D}$. degrees in signal processing and telecommunication from the University of Rennes 1, Rennes Cedex, France, in 1987 and 1990, respectively, and the Habilitation from the University of Nantes, Nantes, France, in 1998.

From 1988 to 1999, he was an Associate Professor with IRESTE-University of Nantes, where he was the Head of the Polarimetry Group, Electronic and Informatic Systems Laboratory. Since 1999, he has been a Full Professor with the University of Rennes 1, where he is currently the Deputy Director of the Institute of Electronics and Telecommunications of Rennes (IETR_CNRS UMR 6164) and also the Head of the Image and Remote Sensing Group-SAPHIR Team. His current research and education activities are centered in the topics of analog electronics, microwave theory, and radar imaging with emphasis in radar polarimetry. His research covers a wide spectrum of areas from radar image processing (SAR, ISAR), polarimetric scattering modeling, supervised/unsupervised polarimetric segmentation, and classification to fundamentals and basic theory of polarimetry. Since 1989, he has been supervising more than 50 research students to graduation (M.Sc. and Ph.D.) in radar polarimetry, covering areas from theory to remote sensing applications. He has coauthored with J.S. Lee (U.S. Naval Research Laboratory) in the topic of polarimetric unsupervised segmentation of POL-SAR data. He has been invited to present 30 presentations in international conferences and 15 in national conferences. He has seven publications in books, 38 papers in refereed journals, and more than 220 papers in conference and symposium proceedings. He has presented advances courses and seminars on radar polarimetry to a wide range of organization and events (DLR, NASDA, JRC, RESTEC, ISAP2000, IGARSS03, EUSAR04, NATO-04, PolInSAR05, IGARSS05, JAXA06, EUSAR06, NATO-06).

Dr. Pottier has chaired and organized 29 sessions in international conferences and was a member of the technical and scientific committees of 17 international symposium or conferences. He was a recipient of the Best Paper Award during EUSAR2000 for his research activities. 